\title{
Mirizzi Syndrome Complicating Acute Cholecystitis: CT Diagnosis
}

\author{
Kassim Sidibé ${ }^{*}$, Pierlesky Elion Ossibi², Zacharia Traoré1, Imane Kamaoui³, \\ Youssef Lamrani' ${ }^{1}$, Meryem Boubbou ${ }^{1}$, Moustapha Maâroufi ${ }^{1}$, Imane Toughrai ${ }^{2}$, \\ Khalid Mazaz', Siham Tizniti1 \\ ${ }^{1}$ Department of Radiology, Hassan II Teaching Hospital, Fes, Morocco \\ ${ }^{2}$ Department of Visceral Surgery, Hassan II Teaching Hospital, Fes, Morocco \\ ${ }^{3}$ Department of Radiology, Mohammed VI Teaching Hospital, Oujda, Morocco \\ Email: "kadiatoulakas@yahoo.fr
}

Received 6 January 2016; accepted 31 May 2016; published 3 June 2016

Copyright (C) 2016 by authors and Scientific Research Publishing Inc.

This work is licensed under the Creative Commons Attribution International License (CC BY). http://creativecommons.org/licenses/by/4.0/

(c) (i) Open Access

\begin{abstract}
Mirizzi syndrome is a rare complication of gallstones with an incidence of less than $1 \%$ per year in Western countries. Imaging and endoscopy play a key role in its diagnosis. CT scan helps to eliminate any malignant lesion of the bile ducts or liver hence comes in handy in the confirmation of Mirizzi syndrome. We hereby report a case of a Mirizzi syndrome complicating acute cholecystitis in a 41-year-old patient without any history of disease.
\end{abstract}

\section{Keywords}

Mirizzi Syndrome, Acute Cholecystitis, CT Scan

\section{Introduction}

Mrizzi syndrome constitutes a rare complication of gallstones. It presents as a case of jaundice due to an extrinsic compression of the common bile duct (CBD): either by an obstructed cystic duct or a calculus obstructing the gallbladder neck. This may lead to either a partial or complete obstruction the bile duct due to underlying local inflammation hence responsible for angiocholitis and could even simulate neoplastic obstructive jaundice. Its incidence varies between $0.7 \%$ and $1.8 \%$ among cholecystectomy patients [1] [2]. We hereby report a case of a Mirizzi syndrome complicating acute cholecystitis in a 41-year-old patient without any history of disease.

${ }^{*}$ Corresponding author.

How to cite this paper: Sidibé, K., Ossibi, P.E., Traoré, Z., Kamaoui, I., Lamrani, Y., Boubbou, M., Maâroufi, M., Toughrai, I., Mazaz, K. and Tizniti, S. (2016) Mirizzi Syndrome Complicating Acute Cholecystitis: CT Diagnosis. Open Journal of Radiology, 6, 92-95. http://dx.doi.org/10.4236/ojrad.2016.62013 


\section{Case Report}

A 41-year-old patient without significant clinical history presented to the emergency ward with jaundice and fever. Symptoms dated back to about 10 days prior to his consultation with the onset of Right upper quadrant (RUQ) pain complicated three days later with jaundice and fever. Physical examination found a $39^{\circ} \mathrm{C}$ febrile patient with $96 \mathrm{HR}$, a clearly visible jaundice and RUQ tenderness.

Laboratory tests showed $13500 / \mathrm{mm}^{3}$ leukocytosis and C-reactive protein level at $148 \mathrm{mg} / \mathrm{l}$, total bilirubin 177; 150 direct bilirubin, Gamma Glutamyl Transferase (GGT): 611; Alkaline phosphatase (ALP): 790; Liver enzymes: Glutamic oxaloacetic transaminase (GOT): 241; glutamic pyruvic transaminase (GPT): 259. The rest of the laboratory tests were unremarkable.

Abdominal ultrasound revealed a stone in the gallbladder with thickened wall and upstream common bile duct and intra hepatic bile ducts dilatation with no visible extra hepatic biliary duct obstacle.

Abdominal CT with contrast medium injection showed a distended gallbladder with thickened wall containing two stones: one enclosed within the cystic duct compressing the common bile duct with an upstream biliary dilatation (Figure 1 and Figure 2).

The patient benefitted from emergent surgery with per-operative discovery of a gallstone stuck in the cystic duct. Cholecystectomy was performed and a Kehr T tube was inserted in the common bile duct. Immediate postoperative recovery was uneventful. The patient was discharged 5 days after surgery with favorable outcome:

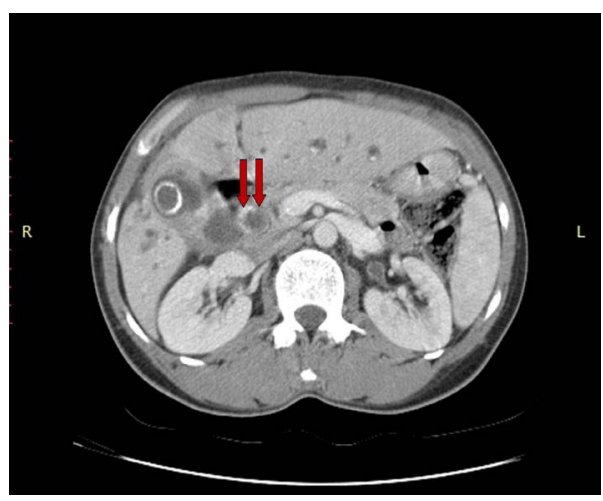

Figure 1. Abdominal CT axial section showing gall stone impaction in the cystic duct and a distended gallbladder with thickened wall containing calculus compressing common bile duct and responsible for upstream bile duct dilatation.

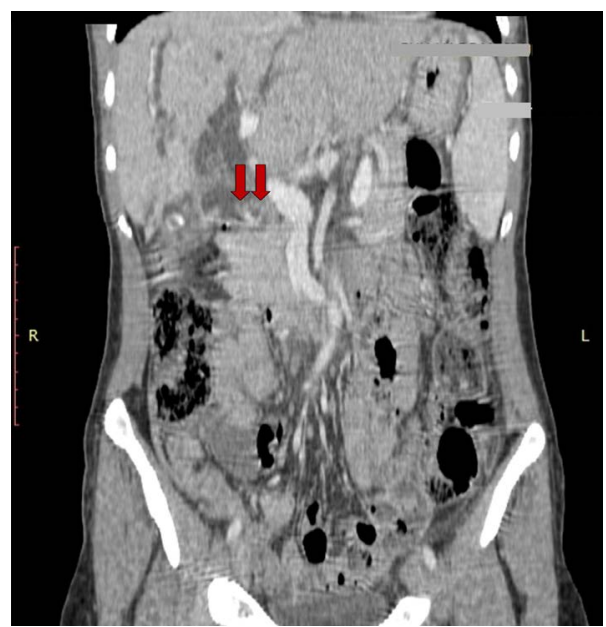

Figure 2. Abdominal CT coronal section after contrast medium injection revealing calculus impaction in the cystic duct with compression of common bile duct and upstream biliary duct dilatation. 
cholangiography through $\mathrm{T}$ tube was performed after 15 days and came back normal. Tube was removed at day 18 after having being clamped for 48 hours.

\section{Discussion}

Mirizzi syndrome is a rare pathological entity in the West with an incidence of less than $1 \%$ per year [3]. However, in developing countries, especially in Latin America, Mirizzi syndrome remains a common occurrence, with an incidence ranging from $4.7 \%$ to 5.7\% [4] [5]. It affects women aged between 53 to 70 years. However this can affect patients of all ages with gallstone [5].

Mirizzi syndrome typically presents as: a long cystic duct parallel to the common hepatic duct and a gallstone impaction in bladder neck or cystic duct resulting in extrinsic compression of common bile duct and subsequent cholangitis.

Mc Sherry in 1982 [6] proposed two variants of the syndrome described as follows

- Type I: simple external compression of the common hepatic duct (typical Mirizzi syndrome);

- Type II: external compression associated with cholecysto-choledochal fistula.

Csendes in 1989 [7], after carefully studying 219 cases, described 4 types

- Type 1: external compression of the common bile duct;

- Type 2: cholecystobiliary fistula affecting less than one-third of the circumference of the bile duct;

- Type 3: fistula involving up to two-thirds of the circumference of the bile duct;

- Type 4: fistula with complete destruction of the wall of the bile duct.

Mirizzi syndrome often presents in an acute form. Chronic forms exist even though clinical presentation remains nonspecific. Typically, it presents as obstructive jaundice (60\% - 100\%), accompanied by right upper quadrant pain (50\% - 100\%) with fever suggestive of acute cholangitis [3].

Laboratory tests reveal an increase in total bilirubin with elevation of liver enzymes notably amino-transferase. In severe cases lab tests may reveal an infectious syndrome.

Preoperative diagnosis of Mirizzi syndrome is made based on imaging and endoscopic investigations.

Ultrasound shows a distended gallbladder with a thickened or normal wall and the presence of oneor more stones in the infundibulum [8]. The common bile duct is dilated with or without intra hepatic duct dilatation [3]. Positive diagnosis of Mirizzi syndrome on ultrasound may be achieved in $29 \%$ of cases [9].

CT may reveal a calculus wedged in the cystic duct. The main utility of computed tomography would be the exclusion of any malignancy of the bile ducts or liver [3]

The MRI clearly shows the presence of gallstones or otherwise in cystic duct and associated dilatation of the common bile duct with or without intra hepatic bile duct dilatation [3]. It is very useful as it is non invasive hence avoiding complications associated with endoscopic cholangiography (ERCP) [10].

Endoscopic retrograde cholangiopancreatography is the first choice imaging technic in its positive diagnosis [6], as it allows more precise anatomical and topographical description of the bile ducts, but is not free of complications such as sepsis and pancreatitis, with a bile duct catheterization failure reportedin $5 \%$ to $10 \%$ of cases [11].

Treatment of Mirizzi syndrome in cases where bile duct is inflamed without biliary fistula (Type I) entails cholecystectomy. Many surgeons contend that laparoscopic cholecystectomy is contraindicated in type VMirizzi syndrome while others consider laparoscopic approach feasible, though technically demanding [12]. Common hepatic duct almost always returns to normal state after the obstructingcalculus has been removed by cholecystectomy and local inflammatory process resolved. Rarely a well-established stricture presents months to years after the acute episode. In such cases, stricture repair of the distal bile duct is indicated for persistent strictures using either Roux-en-Y choledocho-jejunostomy or choledocho-duodenostomy. If cholecysto-choledochal fistula is present, partial cholecystectomy, over sewing the gallbladder cuff and insertion of a T-tube through the fistula is widely regarded as adequate treatment for Type 2 Mirizzi syndrome [13]. Baer et al. [14] suggested the insertion of a T-tube through a separate choledochotomy in the distal CBD in order to prevent excessive leakage and stricture at the fistula site. The presence of CBD stones, fistula, or stenosis at the site of impaction of the stone may necessitate CBD exploration and/or the insertion of a T-tube. Choledocho-duodenostomy for Type 3 forms, and biliary bypass (Roux-en-Y hepatico-jejunostomy) for Type 4 MS are the surgical treatment procedures. In all cases, a frozen section of the gallbladder wall should be done to rule out coexistent cancer.

The prognosis of MS is very favorable for type 1 lesions; as simple cholecystectomy is all that is necessary as 
treatment. Severe types with fistulous destruction of the common duct, postoperative morbidity increases to more than $10 \%$, since biliary fistulae and biliary structuring require dilation or reoperation while liver abscesses may require drainage [15].

\section{Conclusion}

Mirizzi syndrome is a rare entity. Its preoperative positive diagnosis is achieved using ERCP and sometimes, even more rarely so, MRI and CT play a pivotal role alongside the former.

\section{References}

[1] Mishra, M.C., Vashishtha, S. and Tandon, R. (1990) Biliobiliary Fistula: Preoperative Diagnosis and Management Implications. Surgery, 108, 835-839.

[2] Beltran, M.A., Csendes, A. and Cruces, K.S. (2008) The Relationship of Mirizzi Syndrome and Cholecystoenteric Fistula: Validation of a Modified Classification. World Journal of Surgery, 32, 2237-2243. http://dx.doi.org/10.1007/s00268-008-9660-3

[3] Abou-Saif, A. and Al-Kawas, F.H. (2002) Complications of Gallstone Disease: Mirizzi Syndrome, Cholecystocholedochal Fistula, and Gallstone Ileus. The American Journal of Gastroenterology, 97, 249-254. http://dx.doi.org/10.1111/j.1572-0241.2002.05451.x

[4] Corts, M.R. and Vasquez, A.G. (2003) Frequency of the Mirizzi Syndrome in a Teaching Hospital. Cirujano General, 25, 334-337.

[5] Beltran, M.A., Csendes, A. and Cruces, K.S. (2008) The Relationship of Mirizzi Syndrome and Cholecystoenteric Fistula: Validation of a Modified Classification. World Journal of Surgery, 32, 2237-2243. http://dx.doi.org/10.1007/s00268-008-9660-3

[6] McSherry, C.K., Fernstenberg, H. and Virshup, M. (1982) The Mirizzi Syndrome: Suggested Classification and Surgical Therapy. Surgical Gastroenterology, 1, 219-225.

[7] Csendes, A., Diaz, J.C., Burdiles, P., Maluenda, F. and Nava, O. (1989) Mirizzi Syndrome and Cholecystobiliary Fistula: A Unifying Classification. British Journal of Surgery, 76, 1139-1143. http://dx.doi.org/10.1002/bjs.1800761110

[8] Toscano, R.L., Taylor, P.H., Peters, J. and Edgin, R. (1994) Mirizzi Syndrome. The American Journal of Surgery, 60, 889-891.

[9] Safioleas, M., Stamatakos, M., Safioleas, P., Smyrnis, A., Revenas, C. and Safioleas, C. (2008) Mirizzi Syndrome: Mirizzi Syndrome: An Unexpected Problem of Cholelithiasis. Our Experience with 27 Cases. International Seminars in Surgical Oncology, 5, 12. http://dx.doi.org/10.1186/1477-7800-5-12

[10] Lai, E.C.H. and Lau, W.Y. (2006) Mirizzi Syndrome: History, Present and Future Development. ANZ Journal of Surgery, 76, 251-257. http://dx.doi.org/10.1111/j.1445-2197.2006.03690.x

[11] Kim, P.N., Outwater, E.K. and Mitchell, D.G. (1999) Mirizzi Syndrome: Evaluation by MRI Imaging. The American Journal of Gastroenterology, 94, 2546-2550. http://dx.doi.org/10.1111/j.1572-0241.1999.01313.X

[12] Meng, W.C., Kwok, S.P., Kelly, S.B., Lau, W.Y. and Li, A.K. (1995) Management of Mirizzi Syndrome by Laparoscopic Cholecystectomy and Laparoscopic Ultrasonography. British Journal of Surgery, 82, 396. http://dx.doi.org/10.1002/bjs.1800820338

[13] Corlette, M.B. and Bismuth, H. (1975) Biliobiliary Fistula. A Trap in the Surgery of Cholelithiasis. Archives of Surgery, 110, 377-383. http://dx.doi.org/10.1001/archsurg.1975.01360100019004

[14] Baer, H.U., Matthews, J.B., Schweizer, W.P., Gertsch, P. and Blumgart, L.H. (1990) Management of the Mirizzi Syndrome and the Surgical Implications of Cholecystcholedochal Fistula. British Journal of Surgery, 77, 743-745. http://dx.doi.org/10.1002/bjs.1800770708

[15] Waisberg, J., Corona, A. and Abreu, I. (2005) Benign Obstruction of the Common Hepatic Duct (Mirizzi Syndrome): Diagnosis and Operative Management. The Archives of Gastroenterology, 42, 13-18.

http://dx.doi.org/10.1590/s0004-28032005000100005 